\title{
Synthesis of the $\mathrm{C}(1)-\mathrm{C}(12)$ Segment of Peloruside $A$ by an $\alpha$-Benzyloxymethyl Ketone Aldol Strategy
}

\author{
Darren W. Engers, Martin J. Bassindale and Brian L. Pagenkopf*
}

Department of Chemistry and Biochemistry, The University of Texas at Austin, Austin, TX 78712

\section{Supporting Information}

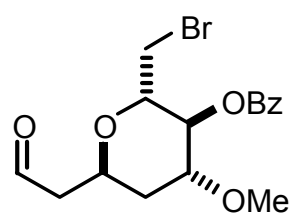

12: $\mathrm{R}_{f} 0.45$ (1:1 EtOAc/hexane); ${ }^{1} \mathrm{H}$ NMR $\left(250 \mathrm{MHz}, \mathrm{CDCl}_{3}\right) \delta 9.83(\mathrm{dd}, J=$ 2.6, $1.7 \mathrm{~Hz}, 1 \mathrm{H}), 8.02(\mathrm{dd}, J=8.3,1.1 \mathrm{~Hz}, 2 \mathrm{H}), 7.56$ (dddd, $J=7.2,7.2,1.4$, $1.4 \mathrm{~Hz}, 1 \mathrm{H}), 7.46-7.39$ (m, 2H), $5.20(\mathrm{dd}, J=3.7,2.2 \mathrm{~Hz}, 1 \mathrm{H}), 4.45$ (ddd, $J=$ $13.1,8.4,4.8 \mathrm{~Hz}, 1 \mathrm{H}), 4.14(\mathrm{dd}, J=7.3,7.3 \mathrm{~Hz}, 1 \mathrm{H}), 3.85(\mathrm{dd}, J=10.7,8.6$ $\mathrm{Hz}, 1 \mathrm{H}), 3.72(\mathrm{dd}, J=10.8,6.3 \mathrm{~Hz}, 1 \mathrm{H}), 3.66(\mathrm{ddd}, J=6.6,3.4,0.0 \mathrm{~Hz}, 1 \mathrm{H})$, 3.45 (s, 3H), 2.68 (ddd, $J=16.5,11.1,2.8 \mathrm{~Hz}, 1 \mathrm{H}), 2.50$ (ddd, $J=16.4,6.1,1.6 \mathrm{~Hz}, 1 \mathrm{H}) 1.90$ $(\mathrm{ddd}, J=14.0,3.4,0.0 \mathrm{~Hz}, 1 \mathrm{H}), 1.82(\mathrm{ddd}, J=9.1,3.6,0.0 \mathrm{~Hz}, 1 \mathrm{H}) ;{ }^{13} \mathrm{C} \mathrm{NMR}(63 \mathrm{MHz}$, $\left.\mathrm{CDCl}_{3}\right) \delta 200.6,165.4,133.4,129.7,129.5,128.5,75.4,74.4,66.8,61.5,57.5,48.6,31.3,29.9$; IR (thin film) $\vee$ 3071-2831, 1720, 1266, 1095, 1068, $711 \mathrm{~cm}^{-1}$; HRMS m/z 371.0502 (calcd for $\left.\mathrm{C}_{16} \mathrm{H}_{18} \mathrm{BrO}_{5}[\mathrm{M}+\mathrm{H}]^{+}, 371.0494\right)$.

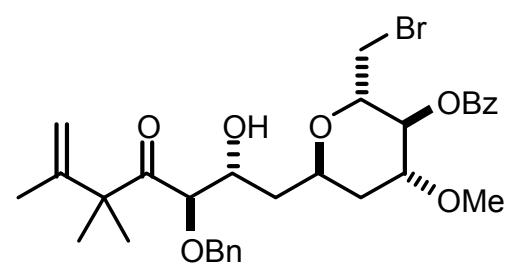

17: $\mathrm{R}_{f} 0.29$ (1:2 EtOAc/hexane); ${ }^{1} \mathrm{H}$ NMR $\left(300 \mathrm{MHz}, \mathrm{CDCl}_{3}\right) \delta$ 8.02 (dd, $J=8.4,1.5 \mathrm{~Hz}, 2 \mathrm{H}), 7.57$ (dddd, $J=7.2,7.2,1.5,1.5$ $\mathrm{Hz}, 1 \mathrm{H}), 7.44(\mathrm{dd}, J=7.8,7.2 \mathrm{~Hz}, 2 \mathrm{H}), 7.33-7.26(\mathrm{~m}, 5 \mathrm{H}), 5.10$ $(\mathrm{dd}, J=3.6,3.0 \mathrm{~Hz}, 1 \mathrm{H}), 5.03(\mathrm{~s}, 1 \mathrm{H}), 5.00(\mathrm{~s}, 1 \mathrm{H}), 4.54(\mathrm{~d}, J=$ $11.4 \mathrm{~Hz}, 1 \mathrm{H}), 4.48(\mathrm{~d}, J=4.2 \mathrm{~Hz}, 1 \mathrm{H}), 4.40(\mathrm{~d}, J=11.1 \mathrm{~Hz}$, $1 \mathrm{H})$, 4.34-4.28 (m, 1H), 4.20 (dddd, $J=9.3,9.3,3.3,3.3 \mathrm{~Hz}$, 1H), 4.06 (ddd, $J=8.1,5.7,3.3 \mathrm{~Hz}, 1 \mathrm{H}), 3.84$ (dd, $J=10.8,9.3$ $\mathrm{Hz}, 1 \mathrm{H}), 3.61$ (dd, $J=8.4,5.1 \mathrm{~Hz}, 1 \mathrm{H}), 3.64-3.58$ (overlapping, m, $1 \mathrm{H}$ ), $3.39(\mathrm{~s}, 3 \mathrm{H}), 2.70$ (d, $J=$ $7.2 \mathrm{~Hz}, 1 \mathrm{H}), 1.85$ (ddd, $J=13.8,9.6,3.3 \mathrm{~Hz}, 1 \mathrm{H}), 1.77-1.66(\mathrm{~m}, 3 \mathrm{H}), 1.68(\mathrm{~s}, 3 \mathrm{H}), 1.30(\mathrm{~s}, 3 \mathrm{H})$, $1.29(\mathrm{~s}, 3 \mathrm{H}) ;{ }^{13} \mathrm{C}$ NMR $\left(75 \mathrm{MHz}, \mathrm{CDCl}_{3}\right) \delta 210.7,165.4,146.3,137.6,133.4,129.7,129.5$, $128.4,128.3,127.9,127.8,113.3,81.7,74.8,74.7,72.1,68.1(2 \mathrm{C}), 63.0,57.3,53.5,36.4,31.8$, $30.9,23.5,23.2,20.4$; IR (thin film) $\vee 3497$ (br), 2969-2332, 1720, 1272, $1107 \mathrm{~cm}^{-1}$; HRMS m/z 603.1935 (calcd for $\mathrm{C}_{31} \mathrm{H}_{39} \mathrm{BrO}_{7}[\mathrm{M}+\mathrm{H}]^{+}$, 603.1957). 


\section{Stereochemical Proof for 3,5-anti:}

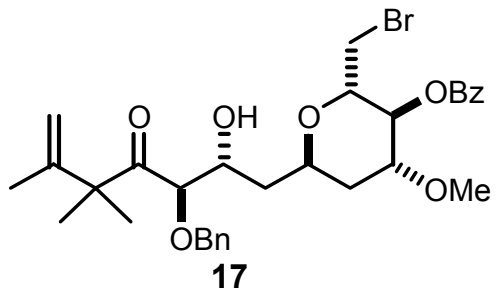<smiles>C=C[C@H](OC(=O)OC)[C@@H](CC1C[C@@H]([C@H](O)C(=O)C(C)(C)C(=C)C)O[Si](C(C)(C)C)(C(C)(C)C)O1)OC</smiles>

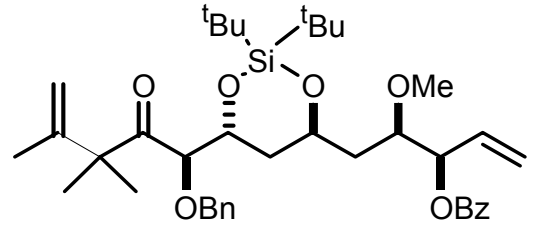

21: $\mathrm{R}_{f} 0.61$ (1:1 EtOAc/hexane); ${ }^{1} \mathrm{H}$ NMR $\left(300 \mathrm{MHz}, \mathrm{C}_{6} \mathrm{D}_{6}\right) \delta$ $8.20(\mathrm{dd}, J=7.2,1.8 \mathrm{~Hz}, 2 \mathrm{H}), 7.23(\mathrm{~d}, J=6.9 \mathrm{~Hz}, 2 \mathrm{H}), 7.12-$ $7.04(\mathrm{~m}, 6 \mathrm{H}), 6.06-5.94(\mathrm{~m}, 2 \mathrm{H}), 5.41-5.33(\mathrm{~m}, 1 \mathrm{H}), 5.10-$ $5.06(\mathrm{~m}, 1 \mathrm{H}), 4.89(\mathrm{~s}, 1 \mathrm{H}), 4.88(\mathrm{~s}, 1 \mathrm{H}), 4.78(\mathrm{ddd}, J=9.0$, $6.3,2.1 \mathrm{~Hz}, 1 \mathrm{H}), 4.66-4.60(\mathrm{~m}, 1 \mathrm{H}), 4.44(\mathrm{~d}, J=11.1 \mathrm{~Hz}$, $1 \mathrm{H}), 4.36(\mathrm{~d}, J=11.4 \mathrm{~Hz}, 1 \mathrm{H}), 4.26(\mathrm{~d}, J=6.3 \mathrm{~Hz}, 1 \mathrm{H}), 3.70$ (ddd, $J=6.3,6.3,4.2 \mathrm{~Hz}, 1 \mathrm{H}), 3.22(\mathrm{~s}, 3 \mathrm{H}), 2.22-2.10(\mathrm{~m}, 2 \mathrm{H}), 1.93-1.83(\mathrm{~m}, 2 \mathrm{H}), 1.60(\mathrm{~s}, 3 \mathrm{H})$, $1.26(\mathrm{~s}, 6 \mathrm{H}), 1.12(\mathrm{~s}, 9 \mathrm{H}), 1.05(\mathrm{~s}, 9 \mathrm{H}) ;{ }^{13} \mathrm{C}$ NMR $\left(75 \mathrm{MHz}, \mathrm{C}_{6} \mathrm{D}_{6}\right) \delta 208.5,165.4,147.0,138.4$, 133.6, 132.9, 130.9, 130.1, 128.6, 128.5, 127.8, 117.5, 112.9, 81.8, 79.2, 74.7, 72.6, 70.0, 68.7, 58.0, 53.6, 38.0, 35.0, 27.4, 27.3, 23.8, 23.2, 21.7, 20.9, 20.5; IR (thin film) v 3096-2857, 1722, $1273,1117 \mathrm{~cm}^{-1}$; HRMS m/z 665.3866 (calcd for $\mathrm{C}_{39} \mathrm{H}_{56} \mathrm{O}_{7} \mathrm{Si}[\mathrm{M}+\mathrm{H}]^{+}, 665.3874$ ). 